\title{
Dietary lipids: less fat or best fat?
}

Jean-Michel CHARDIGNY

\section{Clermont Université,}

Université d'Auvergne,

Unité de Nutrition Humaine,

BP 10448,

F-63000 Clermont-Ferrand,

France;

INRA,

UMR 1019,

Centre de Recherche de Theix,

F-63122 Saint Genes Champanelle,

France

$<$ jean-michel.chardigny@clermont.

inra.fr $>$

Article received 4 December 2012

Accepted 18 December 2012

Obesity is now recognized as a chronic disease that affects the risk of many other diseases such as diabetes, cardiovascular disease, cancer, etc. notably via inflammation.

This overload of body fat is often considered as the result of excess in fat intake, as if the consumption of excess fat

\begin{abstract}
Obesity and overweight occurrence is growing around the word. This is often considered as a consequence of high fat diets, and some recommendations encourage "light" diets, including low fat intake. However, most trials with low fat intake do not demonstrate any benefit and could be worse than low carbohydrate diets. The key role of insulin could explain that eating fat do not make body fat. On the other hand, several unbalanced fatty acid intake are reported, i.e. saturated/mononunsaturated fatty acids and w6/w3 polyunsaturated fatty acids. Thus, fat intake could be improved in this respect. Moreover, the molecular and supramolecular structures of fat in food are new challenges to address in order to ameliorate the recommendations for healthy diets.
\end{abstract}

Key words: lipids, nutrition, obesity, insulin

directly generated the development of fat mass. The result of this shortcut is summed up in a tendency to recommend the "less fat" diet, but forget that the diet is a complex assembly of foods that are vectors of many nutrients including lipids.

However, overweight and obesity affects nearly half of the French population (ObEpi, 2012) with nearly a third of the population overweight (BMI between 25.0 and 29.9) and 15\% of obese people. In addition, the waist size, recognized as an indicator of abdominal fat mass, has on average a steady increase since 1997 (the beginning of the investigation $\mathrm{ObEpi}$ ), with a gain of $3.8 \mathrm{~cm}$ on average for men and $6.8 \mathrm{~cm}$ in women. In 2012, a third of men and $40 \%$ of women have a waist size greater than 102 and $88 \mathrm{~cm}$ respectively, these thresholds being considered as "at risk". The latest OECD data show the same type of development around the world, with $35 \%$ of obese people in the United States in 2010 and projections for 2020
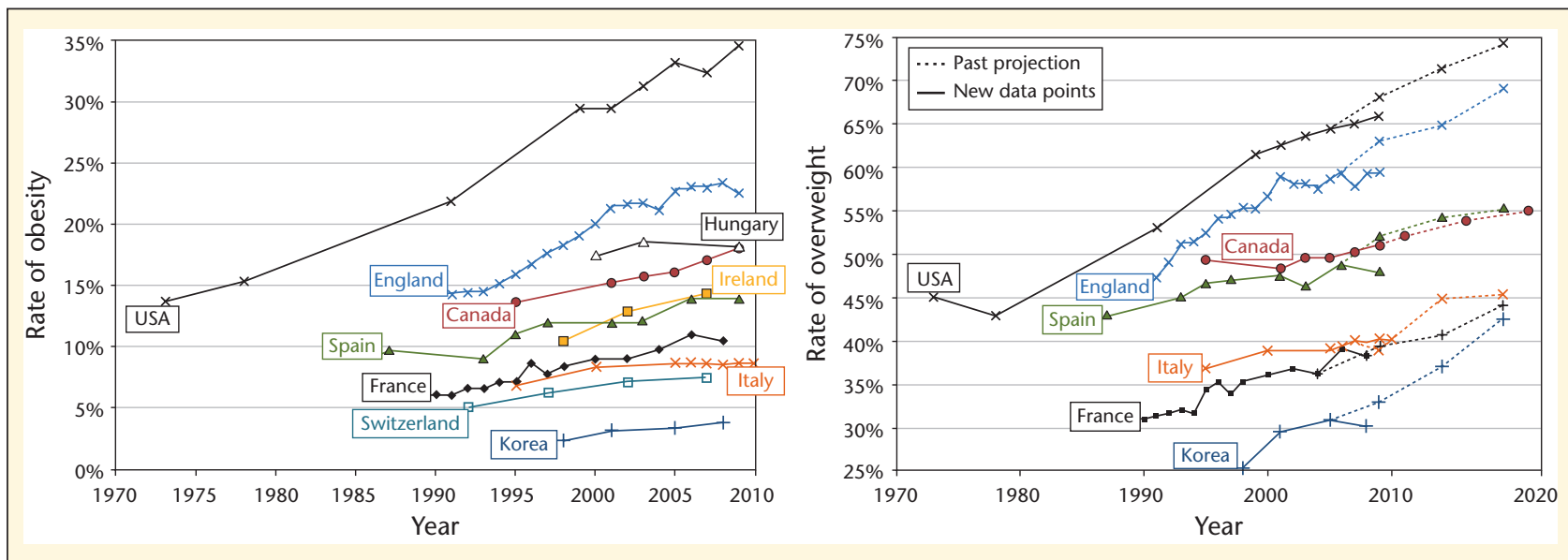

Figure 1. Obesity (left) and overweight (right) evolution since 1970 in different countries (from OECD, 2012).

To cite this article: Chardigny JM. Dietary lipids: less fat or best fat? OCL 2013; 20(2): 71-74. doi : 10.1684/ocl.2012.0498 
in all countries, including Asia (e.g. South Korea) (figure 1).

These tendencies are very disturbing as obesity is now recognized as a risk factor not only for cardiovascular disease or diabetes, but also for kidney or liver diseases, skeletal disorders or muscle loss (sarcopenia).

In France, the average fat intake reported by the INCA surveys are close to the recommendations from ANSES (35-40\% of Total Energy Intake, Recommended Dietary Intake, 2010), although this average masks extreme situations in which fat intake and overall caloric intake is excessive.

In this context, several studies show no benefit related to a reduction in fat intake alone. In 1998, Willett summarized in a paper entitled "/s dietary fat a major determinant of body fat?" that "Diets high in fat do not appear to be the primary cause of the high prevalence of excess body fat in our society, and reductions in fat will not be a solution". This was based on evidence from the discrepancy between the tendency to decrease fat intake between the $70^{\prime}$ and the $90^{\prime}$ and the increase in overweight population in the US (figure 2). Halton et al. (2006) also reported the lack of increase in coronary heart diseases when changing the fat intake from 26.9 to $39.8 \mathrm{En} \%$. The Women's Health Initiative Randomized Controlled Dietary Modifications Trial also showed that the reduction of dietary fat (from 37.8 to 28.8 En $\%)$ did not result in any reduction of cardiovascular or stroke risk in postmenopausal women (Howard et al., 2006). Such results have also been confirmed in intervention trials. As an example, Ebbeling et al. (2007) reported in that a low carbohydrate diet was more beneficial considering HDL and TG plasma concentrations (figure 3).

In this context, insulin is probably one of the key players in the complex metabolic regulation. Insulin is known to facilitate the de novo synthesis of fatty acids from excess circulating glucose and to inhibit the free fatty acid release from adipose tissue. Thus, insulin which plasma concentration is increased after carbohydrate intake but also in insulin resistance situations, could considered as one of the major player to facilitate

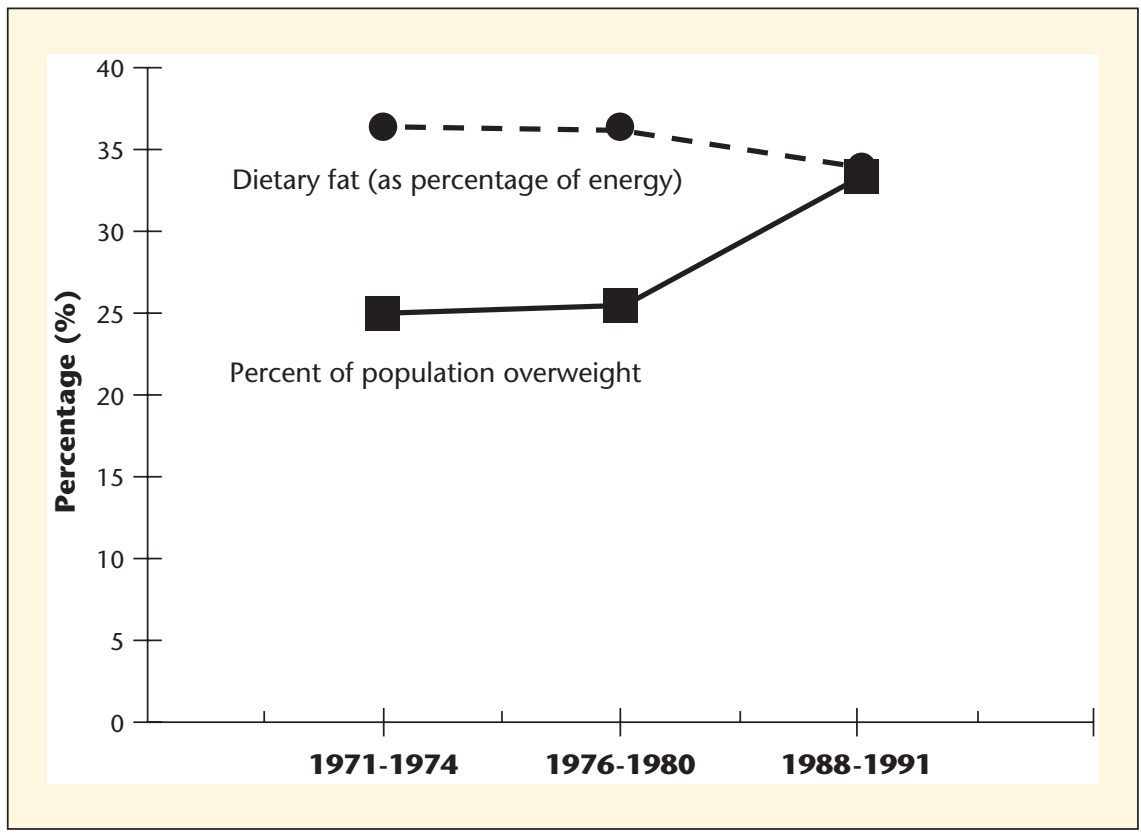

Figure 2. Changes in dietary fat (as percentage of energy) and the percentage of population that is overweight (From Willet, 1998).

\begin{tabular}{|lcc|}
\hline 6 months & Low CHO & Low fat \\
LDL (mg/dL) & $-5,8$ & $-16,3^{*}$ \\
HDL (mg/dL) & $\mathbf{1 , 6}$ & $-4,4^{*}$ \\
TG (\%) & $\mathbf{- 2 1 , 2}$ & $-4,0^{*}$ \\
\hline
\end{tabular}

Figure 3. Plasma lipid changes after 6 months of low CHO or low fat diet (Ebbeling et al., 2007).

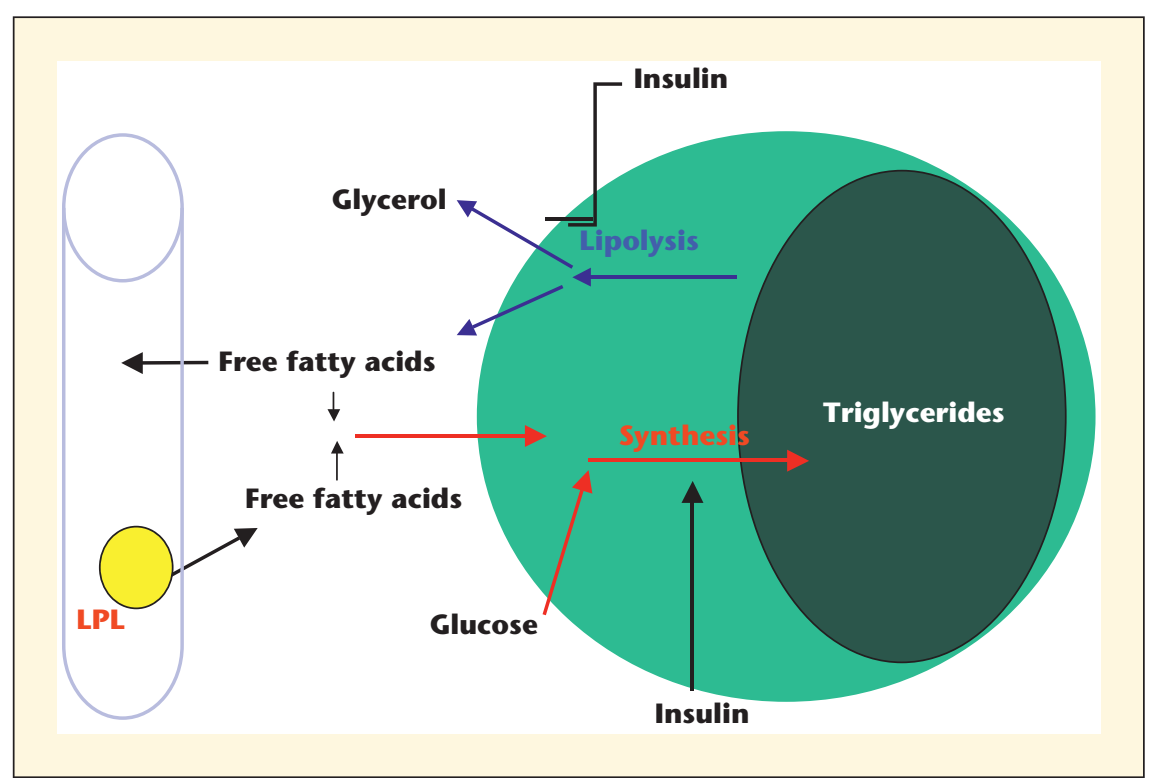

Figure 4. Metabolism of fatty acids in adipose tissue. 
adipose tissue and body fat increase, which means that high carbohydrate diets would be more responsible of excess body fat mass than high fat diets (figure 4).

\section{Thus, if less fat is not the issue, what about best fat?}

Everyone knows that all fatty acids are not only fat an energy, but different classes and subclasses of fatty acids are proposed. When looking at the detailed intake from the INCA2 survey in France, it appears that a first discrepancy between intake and recommendations is the balance between saturates and monounsaturated fatty acids. It is well known that excess of saturates intake is related to an increase in cardiovascular risk, but all the saturated fatty acids cannot be considered as a single bulk. As stated by Mensink et al. (2003) meta-analysis, the different saturated fatty acids do not similarly impact the plasma cholesterol biomarkers (figure 5). It has also been documented that short chain fatty acids have a different metabolic impact than C12C18 SFAs.

The second major unbalance in dietary fatty acids is the excess in omega 6 intake compared to omega-3. This unbalanced has progressed from the middle of the past century and has been clearly demonstrated by Ailhaud et al. by analyzing the fatty acid profile of human milk (figure 6). As the benefit of omega 3 fatty acids in primary prevention of disease is continuously under debate (including alpha-linoleic acid vs EPA and DHA), this unbalance is more clearly documented. However, this questions the bioavailability and sustainability particularly for long chain n-3 fatty acids, but new sourcing like algal production and/or valorisation of fishing by-products could be considered for the future.

Moreover, dietary fatty acids are not present as free fatty acids in the diet. As they are mainly present as triacylglycerols, phospholipids are present in different food items and phospholipids could be a specific carrier to address long chain fatty acids. As an example, Bunea et al. (2004) reported in that $3 \mathrm{~g} / \mathrm{d}$ of krill oil or $3 \mathrm{~g} / \mathrm{d}$ of fish oil did not alter plasma lipids to the same extent. Krill oil (phospholi-

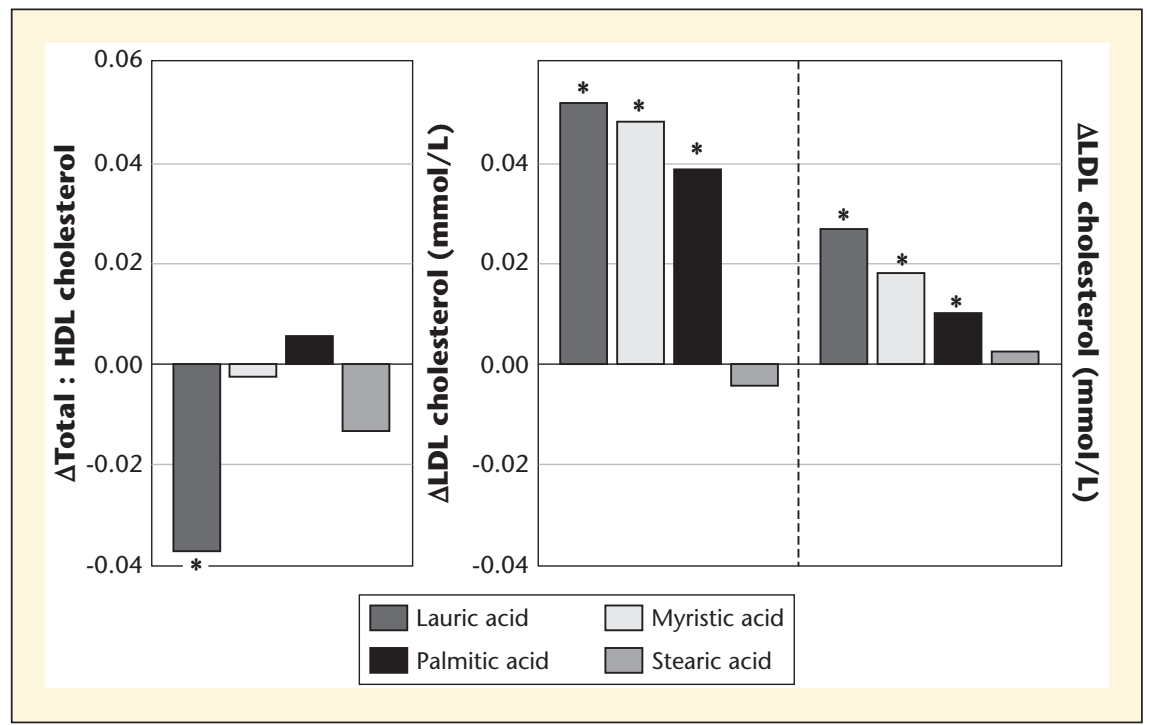

Figure 5. Estimated changes in plasma lipids after 1\% substitution of carbohydrates by different saturated fatty acids (Mensink et al., 2003). pids) was more beneficial on plasma lipids, suggesting specific metabolic trends of PL-esterified fatty acids. This could also be considered for other PL-rich food items as egg yolk.

Another issue to be considered is the supramolecular structure of food items.
Thanks to the LISTRAL network, this issue has recently be reviewed (RaynalLjutovac et al., 2011). Unfortunately, consolidated data in this respect are still lacking and would be helpful for further recommendations which would be more food-based than isolated nutrients-based.

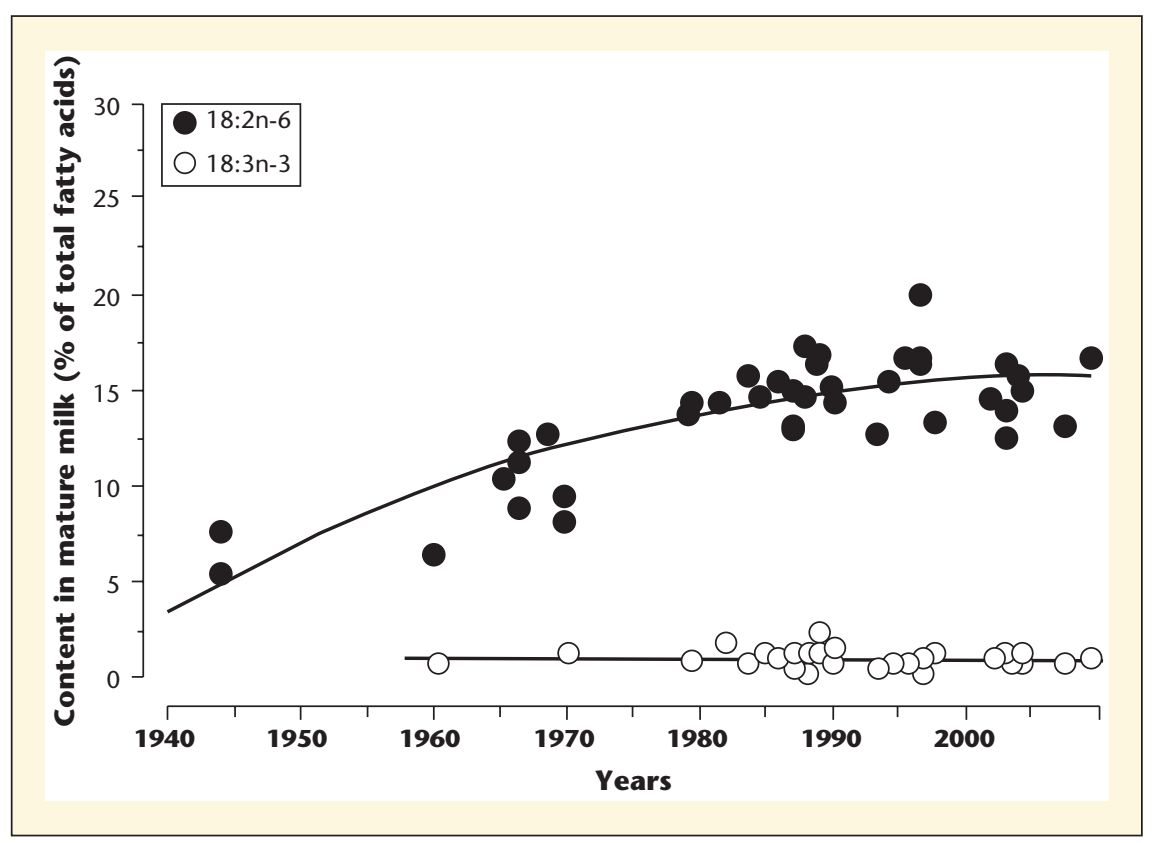

Figure 6. Change in linoleic and a-linolenic acid contents in human milk since the Second World War (Ailhaud et al., 2006). 


\section{Disclosure}

Conflict of interest: none.

\section{REFERENCES}

Ailhaud $G$, Massiera $F$, Weill $P$, Legrand $P$, Alesssandri JM, Guesnet P. Temporal changes in dietary fats: role of $n-6$ polyunsaturated fatty acids in excessive adipose tissue development and relationship to obesity. Prog Lipid Res 2006; 45: 203-36.

Bunea R, El Farrah K, Deutsch L. Evaluation of the effects of Neptune krill oil on the clinical course of hyperlipidemia. Altern Med rev 2004; 9: 420-8.
Ebbeling CB, Leidig MM, Feldma HA, Lovesky MM, Ludwig DS. Effect of a low glycemic load vs low fat diet in obese young adults. A randomized trial. JAMA 2007; 297: 2092102.

Halton TL, Willett WC, Liu S, et al. Low carbohydrate diet score and the risk of coronary heart disease in women. New Engl J Med 2006; 355: 1991-2002.

Howard BV, Va, Horn L, et al. Low-fat dietary pattern and risk of cardiovascular disease. The Women's Health Initiative Randomized Controlles Dietary Modification Trial. JAMA 2006; 295: 655-66.

Mensink RP, Zock PL, Kester ADM, Katan MB. Effects of dietary fatty acids and carbohy- drates on the ration of serum total to HDL cholesterol and on serum lipids and apolipoproteins: a meta-analysis of 60 controlled trials. Am / Clin Nutr 2003; 77: 1146-55.

ObEPi. Enquête épidémiologique nationale sur le surpoids et l'obésité, 60 pages, 2012.

Raynal-Ljutovac K, Bouvier J, Gayet C, et al. Organisation structurale et moléculaire des lipides dans les aliments: impacts possibles sur leur digestion et leur assimilation par I'Homme. OCL 2011; 18: 324-351.

Willett WC. Is dietary fat a major determinant of body fat? Am / Clin Nutr 1998; 67 (Suppl.):556S-62S. 